condition monitoring, sensor integration, tool wear, algorithm

\author{
Christoph RAMSAUER ${ }^{1 *}$ \\ Friedrich BLEICHER ${ }^{1}$
}

\title{
NEW METHOD FOR DETERMINING SINGLE CUTTING EDGE BREAKAGE OF A MULTI-TOOTH MILLING TOOL BASED ON ACCELERATION MEASUREMENTS OF AN INSTRUMENTED TOOL HOLDER
}

\begin{abstract}
In machining applications predominantly for automated machining cells, tool life is often not used to its full extend and cutting tools are exchanged prematurely to avoid tool breakage and thus machine downtime or even damage at work piece or machine. Both effective process monitoring and adequate process control require reliable data from sensors and derived indicators that enable meaningful evaluation. Acceleration measurement by the instrumented tool holder provides signals with high quality from close to the cutting zone. Using the monitoring system, the gained data of the instrumented tool holder can be analyzed especially for the use case of unexpected tool wear, chipping of the cutting edge or breakouts at end mills. This paper describes the data analysis based on the rotational sensor and the corresponding effects on the measurement, an advanced assessment of the spectral distribution in the frequency domain and the experimental results of a test series.
\end{abstract}

\section{INTRODUCTION}

The aim of manufacturers is to produce at low costs parts suitable in quality, whereby tool condition, tool life and using tools to their full potential are relevant factors. To avoid catastrophic failure caused by tool breakage and to guarantee good quality, many tools are not used to their full extend and substituted prematurely. Many options for sensing and evaluating the machine and tool's condition have been published lately [1]. Often surveillance and monitoring systems in terms of digitalization and "Industry 4.0" focus on axis drives' and spindle's power consumption of some kind or vibration measurement within certain parts like bearings or clamping devices $[2,3]$.

The developed a measurement and control system based on an Instrumented Tool Holder [4]. While keeping the tool holder unmodified in its contour, vibration measurement, data transmission and power supply by battery were added. This modified tool holder detects vibration very close to the cutting zone with little influence by the machine's characteristics or the position within the working area. Furthermore, not only online

\footnotetext{
${ }^{1}$ FT - Institute for Production Engineering and Photonic Technologies, TU Wien, Austria

*E-mail: ramsauer@ift.at http://doi.org/10.36897/jme/131918
} 
measurement but also setting countermeasures by adapting cutting parameters or sending feedback to higher level monitoring systems is possible [5]. This system's most prominent applications are drilling and milling operations, especially, for chatter detection and its very successful prevention $[6,7]$.

Further development now leads to even higher sophisticated algorithms to evaluate the raw data of the instrumented tool holder (ITH) in various kinds of ways. While many other possibilities for signal processing mostly rely on statistical features or artificial intelligence [8], the here presented method is based on a model for mechanical vibration and interrupted cut during milling. The focus in this paper is to derive a transparent and causal approach to detect breakouts of single cutting edges while milling based on data of the instrumented tool holder.

\section{MECHANICAL MODEL OF OSCILLATION SYSTEM}

Many already existing measurement systems rely on deflection of strain gauges or piezoelectric components to directly sense and visualize force components. The instrumented tool holder has battery and a wireless Bluetooth transmission system implemented and senses acceleration [9]. The design is depicted in Fig. 1. Thus, a sophisticated mechanical and mathematical model for the ITH to detect irregularities among the cutting edges has to rely on the transducing principles of force excitation and acceleration measurement.

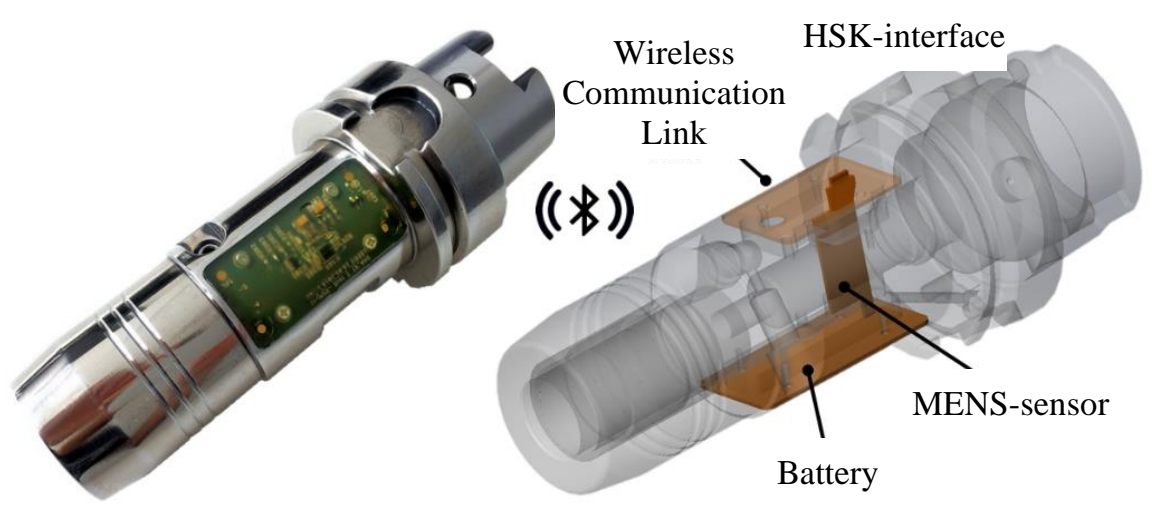

Fig. 1. Sensor integration in tool holder

\subsection{RELATIVE KINEMATICS}

Vibration measurement within the ITH is implemented by an MEMS-Sensor with a vibratory mass. To deduct the results of this paper clearly, relative kinematics is used as general approach for the model of the oscillating spring-mass-damper-system as in Fig. 2. The vibratory mass $m$ primarily moves within the ITH and its frame of reference (colored blue in Fig. 2). The excitation caused by the milling process is mainly relevant in $x$-y-plane 
of the machine tool. Here the point $A$ is the intersection point of the spindle axis. Deviation caused by forces and vibration leads to movement, so that $A$ is not fixed to the inertial system. An easy way of interpretation is to refer to $A$ as the tool center point or at least its corresponding point in the tool holder.

For discussing the stability and vibration of milling processes, it is evident that the most meaningful discussion of vibration will focus on the position vector $\boldsymbol{r}_{A}$ and its derivatives. In the following deduction Newton's second law of motion is applied using the inertial frame of reference. Also $\boldsymbol{r}_{A}$ and its components are given in this frame.

Below, all other vectors are generally written in the basis of the rotating system of the instrumented tool holder as frame of reference that can be seen as the orthogonal main directions of the tool holder and its integrated oscillation system (blue in Fig. 2). The difference in orientation of the systems (inertial system and instrumented tool holder) is modeled by the integration the rotational speed of the spindle $\omega_{S p}$ or rather its integration and a starting angle $\left(\omega_{S p} t+\psi\right)$. Especially the starting angle $\psi$ can be neglected because it can be zero for a suitable starting position and so it is of no further interest.

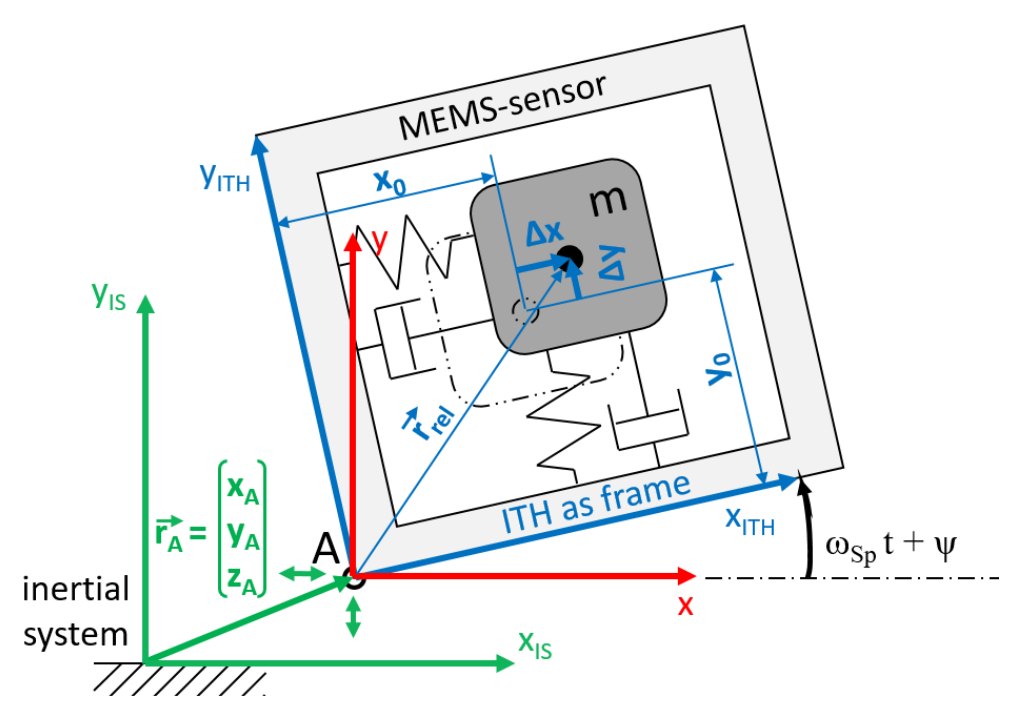

Fig. 2. MEMS-Senor within the tool holder and different frames of reference

Within the instrumented tool holder the center of the vibratory mass $\mathrm{m}$ shall be near $A$, but in general these two points will never be identical, because of finite accuracy of assembly (static eccentricity: $x_{0}, y_{0}$ ) and dynamical effects (additional eccentricity: $\Delta x, \Delta y$ ). Therefore, $\boldsymbol{r}_{r e l}$ refers to the time dependent position of the oscillating mass and it can be described in Eq. 1:

$$
\boldsymbol{r}_{\text {rel|ITH }}=\left[x_{0}, y_{0}, z_{0}\right]^{T}+[\Delta x, \Delta y, \Delta z]^{T}
$$

where: $\boldsymbol{r}_{\text {rel|ITH }}$ - relative position vector to centre of mass $\mathrm{m}$ from $\mathrm{A}$ in tool holder's frame of reference, $\left[x_{0}, y_{0}, z_{0}\right]^{T}$ - static position vector in coordinates of instrumented tool holder, $[\Delta x, \Delta y, \Delta z]^{T}$ - dynamic position vector in coordinates of instrumented tool holder.

The absolute acceleration of the mass $m$ is derived [10] in Eq. 2:

$$
\boldsymbol{a}_{m}=\boldsymbol{a}_{\mathrm{transp}}+\boldsymbol{a}_{\mathrm{rel}}+\boldsymbol{a}_{\mathrm{cor}}
$$


where: $\boldsymbol{a}_{m}-$ vector of absolute acceleration of mass $\mathrm{m}, \boldsymbol{a}_{\text {transp }}-$ vector of transport acceleration, $\boldsymbol{a}_{\text {rel }}-$ vector of relative acceleration, $\boldsymbol{a}_{\text {cor }}-$ vector of Coriolis acceleration.

Inserting all components needed for the kinematic motion leads to Eq. 3:

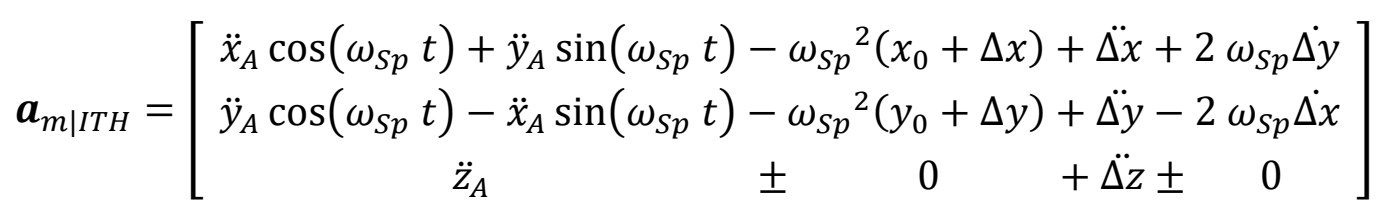

where: $\boldsymbol{a}_{\mathrm{m} \mid I T H}$ - vector of absolute acceleration of mass $\mathrm{m}$ in coordinates of instrumented tool holder, $\left[x_{0}, y_{0}, z_{0}\right]^{T}-$ static position vector, $[\Delta x, \Delta y, \Delta z]^{T}-$ dynamic position vector and its derivatives in time, $\left[x_{A}, y_{A}, z_{A}\right]^{T}$ - position vector of excitation of point $A$ and its derivatives in time in machine's frame of reference, $t$ - time, $\omega_{S p}$ - rotational speed of spindle.

Separating the system's behavior due to rotation into four groups out of Eq. 3 (from left to right), $\boldsymbol{a}_{\mathrm{m} \mid \mathrm{ITH}}$ includes:

- Parts of $A$ 's acceleration $\left[\ddot{x}_{A}, \ddot{y}_{A}, \ddot{z}_{A}\right]$ depending on the angular position,

- Centripetal acceleration caused by spindle's rotational speed and eccentricity,

- Relative acceleration of mass $m$ within the holder,

- Coriolis acceleration of mass m caused by spindle's rotational speed and relative velocity of mass $\mathrm{m}$ within the holder [11].

\subsection{KINETICS AND SYSTEM CHANGE DUE TO ROTATION}

In the simple model of linear springs and dampers, forces substitute these parts easily like in Eq. 4:

$$
m \boldsymbol{a}_{m \mid I T H}=\sum_{i} \boldsymbol{F}_{i}=-\left[\begin{array}{l}
k_{x} \Delta x+c_{x} \dot{\Delta x} \\
k_{y} \Delta y+c_{y} \dot{\Delta y} \\
k_{z} \Delta z+c_{z} \dot{\Delta z}
\end{array}\right]
$$

where: $m$ - vibratory mass, $\boldsymbol{a}_{m \mid I T H}$ - vector of absolute acceleration of mass $\mathrm{m}$ in coordinates of instrumented tool holder, $\boldsymbol{F}_{i}$ - vector $i$ of force $i$ applied on mass $m,[\Delta x, \Delta y, \Delta z]^{T}-$ dynamic position vector and its derivatives in time, $k_{x, y, z}$ - linear spring coefficients in the corresponding direction, $c_{x, y, z}$ - linear damping coefficients in the corresponding direction.

To show the effects of the sensor's rotation clearly, Eqs. 3 and 4 are combined and transformed. The result is a set of three partially coupled differential equations in $[\Delta x, \Delta y$, $\Delta z$ ] with excitation in Eq. 5:

$$
\left[\begin{array}{c}
\ddot{\Delta x}+\dot{\Delta x} \frac{c_{x}}{m}+\Delta x\left(\frac{k_{x}}{m}-\omega_{S p}{ }^{2}\right) \\
\ddot{\Delta y}+\dot{\Delta y} \frac{c_{y}}{m}+\Delta y\left(\frac{k_{y}}{m}-\omega_{S p}{ }^{2}\right) \\
\ddot{\Delta z}+\dot{\Delta z} \frac{c_{z}}{m}+\Delta z\left(\frac{k_{z}}{m}\right)
\end{array}\right]=\left[\begin{array}{c}
-\ddot{x}_{A} \cos \left(\omega_{S p} t\right)-\ddot{y}_{A} \sin \left(\omega_{S p} t\right)+\omega_{S p}{ }^{2} x_{0}-2 \omega_{S p} \dot{\Delta y} \\
-\ddot{y}_{A} \cos \left(\omega_{S p} t\right)+\ddot{x}_{A} \sin \left(\omega_{S p} t\right)+\omega_{S p}{ }^{2} y_{0}+2 \omega_{S p} \dot{\Delta x} \\
-\ddot{z}_{A}
\end{array}\right]
$$


where: $m$ - vibratory mass, $[\Delta x, \Delta y, \Delta z]^{T}$ - dynamic position vector and its derivatives in time, $\left[x_{\mathrm{A}}, y_{\mathrm{A}}, z_{\mathrm{A}}\right]^{T}$ - position vector of excitation of point $A$ and its derivatives in time written in machine's frame of reference, $\left[x_{0}, y_{0}, z_{0}\right]^{T}-$ static position vector, $t$-time, $\omega_{S p}$ - rotational speed of spindle, $k_{x, y, z}$ - linear spring coefficients in the corresponding direction, $c_{x, y, z}$ - linear damping coefficients in the corresponding direction.

Considering the systems tuning and excitation, it can be concluded from the left-hand side of the Eq. 5:

- Spindle rotation reduces the system's stiffness and misadjusts the sensor's internal tuning to a lower natural frequency, similar to shaft bending modes in turbomachinery [12].

- Normally the acceleration sensor as purchased MEMS-part is assembled onto nonrotating systems. This leads to its specifications in the data sheet. It is wise to choose the sensor's natural frequency much higher than the rotational speed.

The right-hand side of Eq. 5 shows:

- The effect of nonlinear modulation due to rotation in $\mathrm{x}$ and $\mathrm{y}$ [13]: Considering that certain excitation frequencies cause $A$ 's acceleration $\left(\ddot{x}_{A}, \ddot{y}_{A}\right)$, only mixture products with trigonometric functions occur in the rotational sensor's excitation. This causes other frequencies to be detected in ITH than in stationary sensors [14].

- A constant offset in $\mathrm{x}$ and $\mathrm{y}$ due to eccentric assembly. To lower this influence either spindle speed needs to be limited (as it has quadratic influence), or the assembly needs to be as precise as possible.

- Coupling of $x$ and $y$ direction caused by Coriolis effect.

\subsection{EXPECTED EXCITATION}

The excitation $\left(\left[\ddot{x}_{A}, \ddot{y}_{A}, \ddot{z}_{A}\right]^{T}\right)$ of the milling process can be categorized into three main groups when using tools with regular pitch and number of cutting edges $z$. These categories are also illustrated in a generic spectrum in Fig. 3.

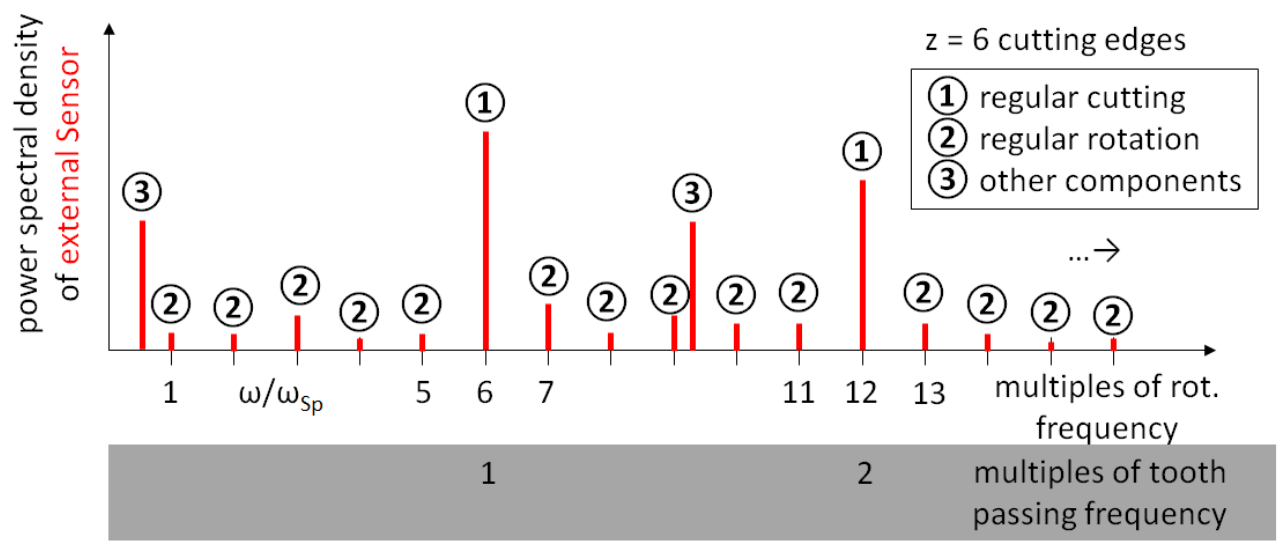

Fig. 3. PSD of milling machines' vibration sensed by external (fixed) sensors

(1) Frequency-terms that occur with $\omega=k z \omega_{S p}(k \in N)$ are associated with the main cutting process and the generally regular chipping of the material. These components are expected to occur mainly when processing under good and stable conditions $[15,16]$. 
(2) Frequency-terms that occur with $\omega=k \omega_{S p}(k \in N)$ include the first group but do also content all other effects resulting from the spindle spinning, such as concentricity errors and geometrical deviations from the tool and roller bearing defects. In general, these terms describe all time signals repeating periodically with the rotation of the spindle.

(3) All other frequencies may contain stimulations form outside the machine or other parts inside the machine, also chatter frequencies. Furthermore, this group generally contains natural frequencies of all kinds of structures within the machine.

\subsection{MEASUREMENT CHAIN IN FREQUENCY DOMAIN}

As in Eq. 3 already anticipated, the modulation scheme in the time domain according to can be written in Eq. 6 [13]:

$$
\ddot{\mathrm{r}}_{\mathrm{A} \mid \mathrm{ITH}}(\mathrm{t})=\underline{\mathrm{A}}(\mathrm{t}) \ddot{\mathrm{r}}_{\mathrm{A} \mid \mathrm{IS}}(\mathrm{t})=\left[\begin{array}{ccc}
\cos \left(\omega_{S p} \mathrm{t}\right) & \sin \left(\omega_{S p} \mathrm{t}\right) & 0 \\
-\sin \left(\omega_{S p} \mathrm{t}\right) & \cos \left(\omega_{S p} \mathrm{t}\right) & 0 \\
0 & 0 & 1
\end{array}\right]\left[\begin{array}{c}
\ddot{x}_{\mathrm{A}}(\mathrm{t}) \\
\ddot{y}_{\mathrm{A}}(\mathrm{t}) \\
\ddot{z}_{\mathrm{A}}(\mathrm{t})
\end{array}\right]
$$

where: $\ddot{\boldsymbol{r}}_{\mathrm{A} \mid \mathrm{ITH}}$ - tool holder's acceleration in time domain and in tool holder's frame of ref., $\ddot{\boldsymbol{r}}_{\mathrm{A} \mid \mathrm{IS}}$ - tool holder's acceleration in time domain and in machine's frame of reference, $\underline{\boldsymbol{A}}$-rotational transformation matrix, $\left[\ddot{x}_{A}, \ddot{y}_{A}, \ddot{z}_{A}\right]^{T}$ - vector of acceleration of point $A$ in time domain in machine's frame of reference, $t$ - time, $\omega_{S p}$ - rotational speed of spindle.

In the frequency domain this scheme results in a nonlinear modulation in $X$ and $Y$ due to the multiplication with time-dependent trigonometric functions in the time domain [14]. So, the spectral information of the tool holder's acceleration in the inertial system $\left(\ddot{\boldsymbol{R}}_{\mathrm{A} \mid \mathrm{IS}}\right)$ is modified by modulation.

$$
\ddot{\mathrm{R}}_{\mathrm{A} \mid \mathrm{ITH}}=\left[\begin{array}{c}
\ddot{\mathrm{X}}_{\mathrm{A}}\left(\omega-\omega_{\mathrm{Sp}}\right) \\
\ddot{\mathrm{X}}_{\mathrm{A}}\left(\omega+\omega_{\mathrm{Sp}}\right) \\
\ddot{\mathrm{Y}}_{\mathrm{A}}\left(\omega-\omega_{\mathrm{Sp}}\right) \\
\ddot{\mathrm{Y}}_{\mathrm{A}}\left(\omega+\omega_{\mathrm{Sp}}\right) \\
\ddot{\mathrm{Z}}_{\mathrm{A}}(\omega)
\end{array}\right]=\frac{1}{2}\left[\begin{array}{ccc}
1 & -\mathrm{i} & 0 \\
1 & \mathrm{i} & 0 \\
\mathrm{i} & 1 & 0 \\
-\mathrm{i} & 1 & 0 \\
0 & 0 & 2
\end{array}\right]\left[\begin{array}{c}
\ddot{\mathrm{X}}_{\mathrm{A}}(\omega) \\
\ddot{\mathrm{Y}}_{\mathrm{A}}(\omega) \\
\ddot{\mathrm{Z}}_{\mathrm{A}}(\omega)
\end{array}\right]=\mathrm{f}\left(\ddot{\mathrm{R}}_{\mathrm{A} \mid \mathrm{IS}}(\omega)\right)
$$

where: $\ddot{\boldsymbol{R}}_{\mathrm{A} \mid \mathrm{ITH}}$ - tool holder's acceleration in frequency domain and in tool holder's frame of ref., $\ddot{\boldsymbol{R}}_{\mathrm{A} \mid \mathrm{IS}}$ - tool holder's acceleration in frequency domain and in machine's frame of reference, $\left[\ddot{X}_{A}, \ddot{Y}_{A}, \ddot{Z}_{A}\right]^{T}$ - vector of acceleration of point $\mathrm{A}$ in frequency domain in machine's frame of reference, $i$ - imaginary unit, $\omega$ - frequency, $\omega_{s p}$ - rotational speed of spindle

The relation for the amplitudes (or for the power spectral density PSD) can be written as a shift of the excitation frequencies $\omega$ resulting in "disintegrated" frequencies $\left(\omega \pm \omega_{S p}\right)$. With an excitation at a given frequency in the inertial system (IS), the resulting signal in frame of reference of the ITH is derived theoretically as in Eq. 8 [15]. This effect is caused by the constant rotational speed $\omega_{S p}$. Even Coriolis acceleration just couples $x$ and $y$ direction but will not create further components beside these frequencies. 


$$
\left[\begin{array}{c}
\ddot{X}_{A}(\omega) \\
\ddot{Y}_{A}(\omega) \\
\ddot{Z}_{A}(\omega)
\end{array}\right]_{\mid I S} \mapsto\left[\begin{array}{c}
\ddot{X}_{A}\left(\omega \pm \omega_{S p}\right) \\
\ddot{Y}_{A}\left(\omega \pm \omega_{S p}\right) \\
\ddot{Z}_{A}(\omega)
\end{array}\right]_{\mid I T H}
$$

where: $\left[\ddot{X}_{A}, \ddot{Y}_{A}, \ddot{Z}_{A}\right]^{T} \mid I S$ - vector of acceleration of point A in frequency domain in machine's frame of reference, $\left[\ddot{X}_{A}, \ddot{Y}_{A}, \ddot{Z}_{A}\right]^{T} \mid I T H$ - vector of acceleration of point $A$ in frequency domain in tool holder's frame of reference, $\omega$ - frequency, $\omega_{S p}$ - rotational speed of spindle.

\section{DERIVED MODEL FOR DETECTION OF UNEVEN WEAR IN MILLING OPERATIONS}

Combining knowledge of the modulation of Eq. 8 and the excitation (Section 2.3), an indicator for the regularity of cutting out of the data of the instrumented tool holder is processed. The percentage of power caused by regular cutting $\left(\omega=k z \omega_{S p} ; k \in N\right)$ in relation to all effects caused by spindle rotation $\left(\omega=k z \omega_{S p} ; k \in N\right)$ is quantified in Eq. 9 with respect to the shift of frequency terms. Squaring the amplitudes values leads to a quotient that is closely connected to the power spectral density. The here shown quotient only considers information of one radially aligned measurement direction (e.g. $x$ ). The same procedure can be used for every other radial measurement direction (e.g. $y$ ).

$$
Q_{\text {Theory }}=\frac{\sum_{k}\left|X_{I T H, k}\left((k z \pm 1) \omega_{S p}\right)\right|^{2}}{\sum_{k}\left|X_{I T H, k}\left(k \omega_{S p}\right)\right|^{2}}
$$

where: $Q_{\text {Theory }}$ - quality index according to theory, $k$ - natural number as counter, $X_{I T H, k}-$ instrumented tool holder's signal in frequency domain in $x$ direction evaluated at certain frequencies, $z$ - number of cutting edges, $\omega_{S p}$ - rotational speed of spindle.

So, the amplitudes or spectral power components can be summed selectively. For instance a distribution like on the left side of Fig 4 would lead to $Q=1$ (best condition possible) and the shown distribution on the right side may be around $Q=0.6$ (rather bad condition).

For a reliable evaluation of real measurement data the mere summation of discrete components is widened to an area of interest $( \pm \Delta \omega)$, in which the power spectral density gets integrated. This results in a more robust algorithm. Also a maximum order of tooth passing frequency $(j)$ is introduced. However, only components up to a certain maximum frequency are considered and evaluated. For the case of a milling tool with three or more cutting edges, Eq. 10 shows the general definition of the quality index $Q$, that quantifies the share of acceleration due to regular cutting relatively to all acceleration periodic with spindle's rotation:

The highlighted areas on the left subfigure of Fig. 5 correspond with the elements in Eq. 10. As can be seen on the right side of Fig. 5, this procedure needs to be adapted for tools with two cutting edges. In this case, modulation products of neighboring frequencies interact. 

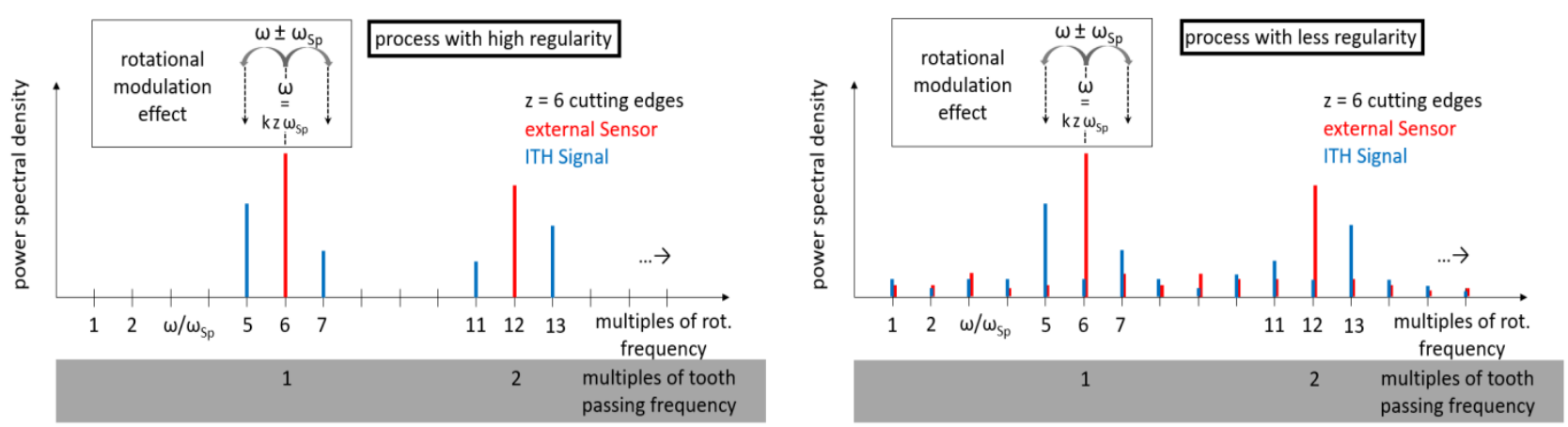

Fig. 4. PSD of milling machine's vibration sensed by fixed sensors (red) and instrumented tool holder (blue)

$$
Q_{\text {Algorthim }}(\mathrm{j}, \Delta \omega)=\frac{\sum_{k=1}^{j}\left(\int_{(k z-1) \omega_{S p}}^{(k z-\Delta \omega} p^{-\Delta \omega} p s d(\omega) d \omega+\int_{(k z+1) \omega_{S p}-\Delta \omega}^{(k z+1) \omega_{S p}+\Delta \omega} p s d(\omega) d \omega\right)}{\sum_{k=1}^{j z+1} \int_{k \omega_{S p}-\Delta \omega}^{k \omega_{S p}+\Delta \omega} p s d(\omega) d \omega}
$$

where: $Q_{\text {Algorithm }}$ - quality index for implementation in algorithm, $j$ - natural number as counter, $k$ - natural number as counter, $\Delta \omega-$ comb width, $p s d$ - power spectral density of instrumented tool holder's signal evaluated at certain frequencies, $z$ - number of cutting edges, $\omega_{S_{p}}$ - rotational speed of spindle, $\omega$ - frequency
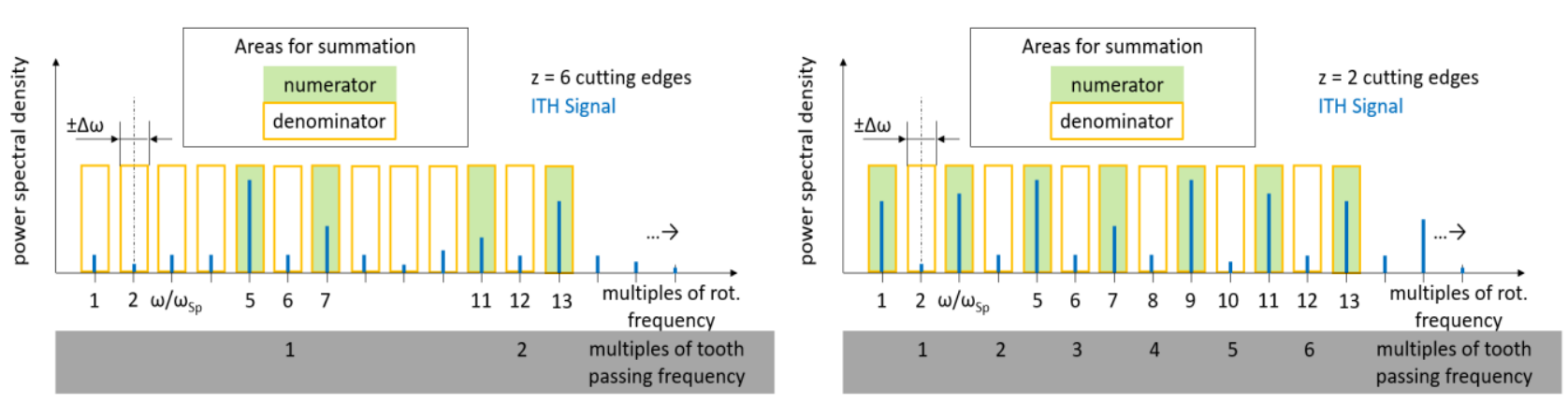

Fig. 5. PSD of processes with moderate regularity: highlighted areas for tool condition evaluation based on the number of cutting edges (left: $z=6$; right: $z=2$ ), sensed by the instrumented tool holder (blue)

Eventually, the quality index $Q$ can be implemented as an algorithm for both postprocessing data evaluation and online-monitoring. To validate this theoretically developed quotient and its power for process evaluation, several test series have already been carried out.

\section{TEST SERIES}

Based on theory, the quality index $Q$ detects unevenness and irregularities of milling processes, caused by effects occurring at a single cutting insert out of many. This procedure has already been proven useful for local events of cutting edge chipping [9]. In general, all forms of uneven wear situations can be addressed. 


\subsection{TEST SETUP FOR CUTTING EDGE BREAKOUT}

In the here presented test setup, a milling head of diameter $25 \mathrm{~mm}$ with 4 cutting inserts was clamped in the instrumented tool holder. Milling aluminum along a straight path of $100 \mathrm{~mm}$ in a DMG Mori DMU 75 monoBlock, the data signals of the instrumented tool holder were recorded and evaluated using the quality index $Q$. Detailed process parameters are listed within Fig. 6. One of four cutting inserts was exchanged (conditions C1-C2-C3), while the other three cutting edges remained at condition $\mathrm{C} 1$. In each of these three setups, three cuts were performed under the same conditions. Conditions C2 and C3 were artificially generated by improper handling of the cutting insert leading to heavy outbreaks. So, the setup simulated a situation right after a spontaneous and local outbreak on one cutting insert, while the other inserts were still in good condition (C1).

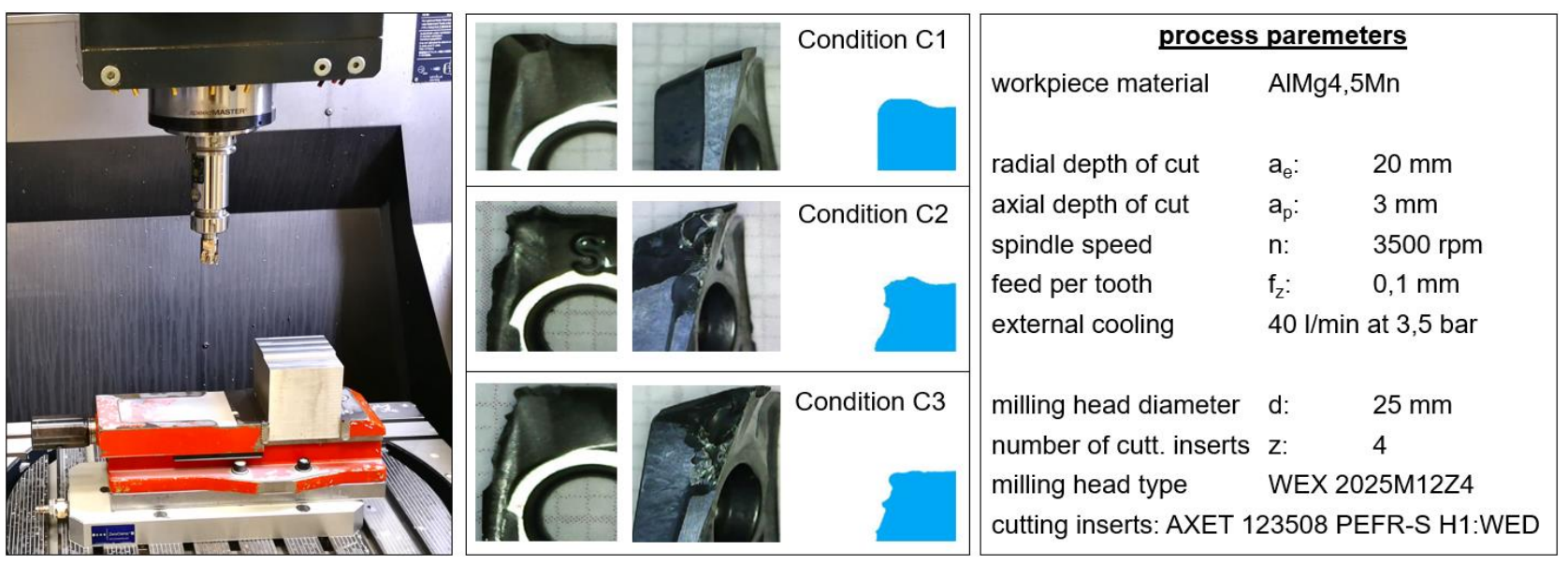

Fig. 6. Experimental setup in DMG Mori DMU 75 (left), details of cutting edge condition (center), process parameters (right)

\subsection{RESULTS}

The used tool holder provides acceleration measurement in three orthogonal axes (two radially aligned and one in axial direction). For the following results, the signals of the two radial directions ( $X$ and $Y$ ) were evaluated separately using cuts of the signal of 1.5 seconds during the milling operation.

As in Fig. 7, the found algorithm can distinguish the changed condition of a single cutting insert sharply. The quality index $Q$ (as defined in Eq. 10) of each radial direction separates the condition C1 clearly, but an even better picture can be drawn when plotting $Q_{y}$ over $Q_{x}$. Similar results have also been achieved when evaluating sections where the path of milling included former grooves and drill holes. Monitoring the tool's condition and detecting heavy spontaneous tool wear was possible even before the final work piece surface had been affected, as illustrated in Fig. 8. Also the detection of the wear situation is possible, before a rapidly escalating state of wear effects the next cutting insert following the initially worn one due to overload. 


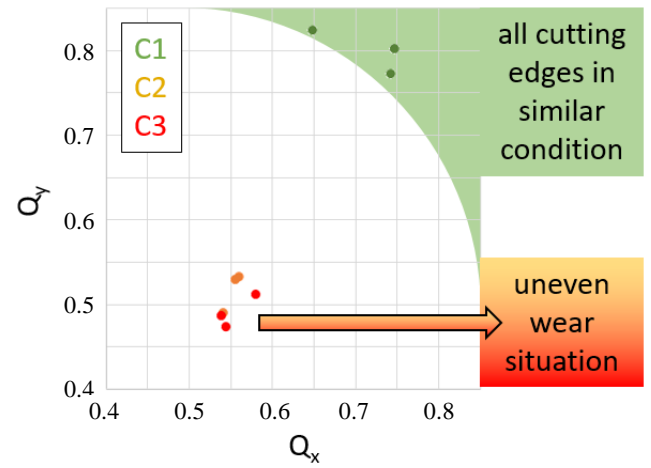

Fig. 7. Results of cutting tests: two radial quality indices plotted as $Q_{y}$ over $Q_{x}$
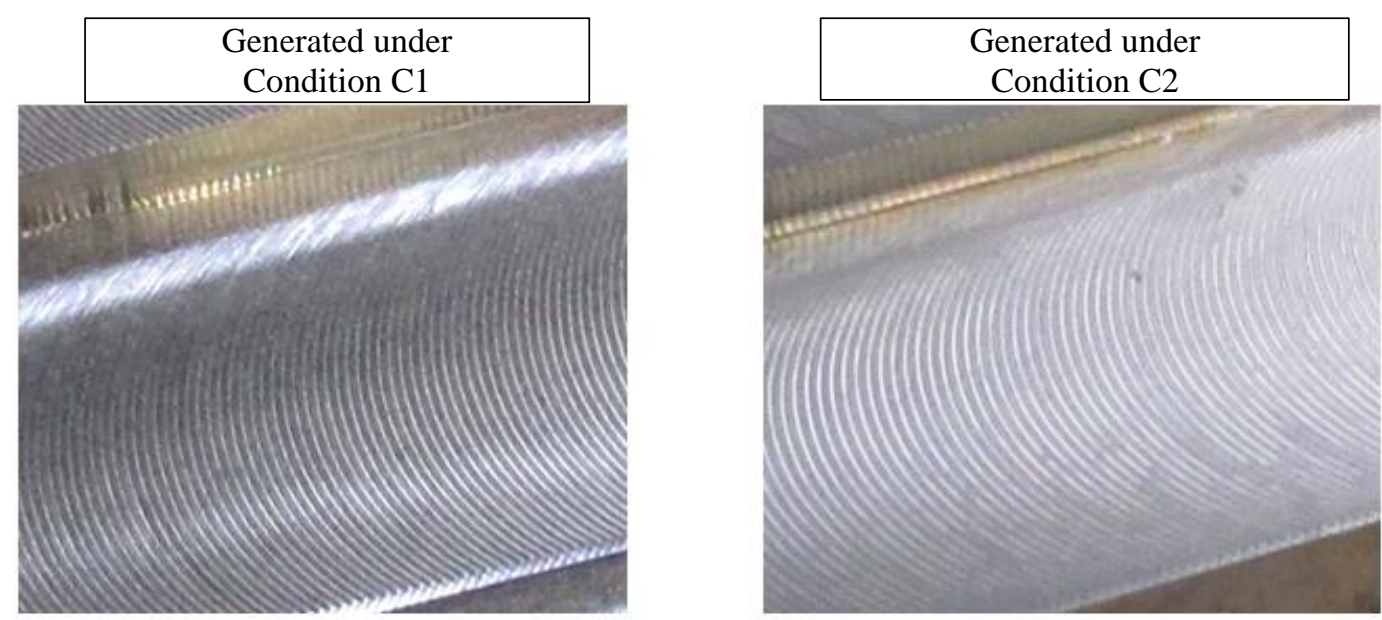

Fig. 8. Generated surface

\section{CONCLUSION}

In this paper an algorithm for detection of spontaneous tool wear has been derived based on theoretical considerations. Designed for the acceleration sensitive instrumented tool holder, the quality index quantifies the percentage of power due to regular cutting in relation to the acceleration signal's power coming along with spindle's rotation. This leads to a quotient suitable for detection of uneven tool wear situation like outbreaks or chipping and gives the possibility to monitor and interact even before the work piece quality is affected.

The instrumented tool holder applies measurement functionality even in the tough environment of well-adapted production processes. This paper derives a basis for data evaluation that can be executed on-line in a monitoring and control system. When detecting the breakout, the control system can interact at a crucial point where only one cutting edge has faced breakouts. For instance, reducing feed to its half also reduces the load on the following cutting edge and helps to avoid an escalating effect. So, tool costs can decrease if every tool is used to its individual end of lifetime by detecting severe breakage or its approach. 


\section{ACKNOWLEDGEMENTS}

TU Wien as well as the authors want to express their appreciation and thanks to the Machine Tool Technologies Research Foundation (MTTRF) for supporting the research work.

\section{REFERENCES}

[1] GOETZ S., 2020, Process Monitoring in End Milling Using Polar Figures, J. Mach. Eng., 20/3, 95-105.

[2] ZHANG X.Y., 2018, A Multi-Sensor Based Online Tool Condition Monitoring System for Milling Process, Procedia CIRP 72, 1136-1141.

[3] UHLMANN E., LAGHMOUCHI A., GEISERT C., HOHWIELER E., 2017, Smart Wireless Sensor Network and Configuration of Algorithms for Condition Monitoring Applications, J. Mach. Eng., 17/2, 45-55.

[4] BLEICHER F., SCHÖRGHOFER P., HABERSOHN C., 2018, In-Process Control with a Sensory Tool Holder to Avoid Chatter, J. Mach. Eng., 18/3, 16-27.

[5] SCHÖRGHOFER P., PAUKER F, LEDER N., MANGLER J., RAMSAUER CH., BLEICHER F., 2019, Using Sensory Tool Holder Data for Optimizing Production Processes, J. Mach. Eng., 19/3, 43-55.

[6] https://schunk.com/at_en/homepage/itendo/ (accessed 24 Sept 2020).

[7] https://www.mytoolit.com/ICOtronic/ (accessed 24 Sept 2020).

[8] JEMIELNIAK K., 2019, Contemporary Challenges in Tool Condition Monitoring, J. Mach. Eng., 19/1, 48-61.

[9] BLEICHER F. 2020, Method for Determining Edge Chipping in Milling Based on Tool Holder Vibration Measurements, CIRP Annals, 69, 101-104.

[10] AWREJCEWICZ J., 2012, Classical Mechanics - Kinematics and Statics, Springer.

[11] JAIN S.R., 2016, Mechanics, Waves and Thermodynamics, Cambridge University Press, New York.

[12] CHILDS D., 1993, Turbomachinery Rotordynamics - Phenomena Modeling and Analysis, John Wiley \& Sons Inc., New York.

[13] POWERS J.M., 2015, Mathematical Methods in Engineering, Cambridge University Press, Cambridge.

[14] BEERENDS R.J., 2003, Fourier and Laplace Transforms, Cambridge University Press, Cambridge.

[15] SCHMITZ T.L., 2019, Machining Dynamics - Frequency Response to Improved Productivity, Springer Nature Switzerland AG, Cham.

[16] BALACHANDRAN B., 2001, Nonlinear Dynamics of Milling Processes, Philosophical Transactions: Mathematical, Physical and Engineering Sciences, 359/1781, 793-819. 